\title{
Performance Analysis of a Cut-through vs Packet-switching Techniques
}

\author{
N. M. A. AYAD and F. A. MOHAMED \\ Reactor Dept., Computer Lab., Atomic Energy Establishment, \\ Atomic Energy Post Office Box No. 13759, EGYPT.
}

\begin{abstract}
A simulation package containing a class of five adaptive routing algorithms has been used to investigate the performance of the virtual cut-through switching technique. The five algorithms are: the shortest path, the learning automata, the deterministic rule, the distributed compulations and the relaxation principle routing algorithms. Performance criteria include the average delav, average transit time, average retransmission probability and network utilization. These parameters are intimately related to each other. They are compared with that of the packet switching using the same routing algorithms. lifficient switching techniques guarantee the best value for these performance parameters. The results obtained will be an aid in comparing the two switching techniques under different routing algorithms.
\end{abstract}

\section{Introduction}

The backbone of any flow control is the routing algorithm. The routing algorithm characterizes the packet delay, the packet transit time through the network, the buffer management and size, etc. On the other hand, the switching technique determines the network cost functions, typically the network response time, the estimated delays, the throughput, the probability of retransmission, and the network utilization [7,13].

In attempting to use the computer networks more effectively and more powerfully, adaptive routing algorithms have been favored due to their high reliability and efficiency. These algorithms attempt to maintain the optimal assignments of routes for the network traffic [12].

There are two basic data switching techniques, the circuit switching and the store-and-forward. The various needs of data rates, messages length and message arrival rates have led to the concept of integrated (or hybrid) networks that include more than one switching technique. If the message to be transmitted is very long, it may be cost effective to transmit it over dedicated path as in the case with circuit switching. If it is relatively short and infrequently occurring, a message switching facility is suitable, particularly with message from different users being combined to share the transmission costs [3]. Modern time switching and time multiplexing technology allow both modes of operation to be incorporated in one system.

One of these techniques in that direction is the virtual cut-through switching [9]. In this technique, a message is not stored fully at an intermediate node even if the node is free. The message header (xo), however has to be processed in order to choose the next outgoing channel for that particular message. The message header contains the information on routing and addressing. It means that the message can start its onward journey through the network immediately after its header has been processed. When this happens the message is said to have made a full cut.

This however, happens only when the intermediate node(s) is(are) found empty by an arriving message. Otherwise, the modified virtual cut-through allows partial cuts. The idea of a partial cut is motivated by the fact that, when an arriving message finds the node busy servicing the only packet present at the node, the newly arrived packet does not have to be fully stored at that particular node and can start its onward journey as soon as the preceding message's service finished. Gain in delay per message for a partial cut is of course less than that in the case of full cuts [8]. In this work we are considering only the case of full cuts.

This paper presents the performance analysis of a class of five adaptive routing algorithms under a Virtual Cutthrough (VC) as compared to the conventional Packet Switching (PS) technique. These routing algorithms are the Shortest Path (SP), the Learning Automata (LA), the Deterministic Rule(DR), the Distributed Computation (DC) and the Relaxation Principle (RP). At any time just one of these algorithms should be applied which is typical for an actual network. The overall average time delay, the average transit time, the average retransmission 
a common basis for the comparative study. Section 2 discusses the switching techniques. Section 3 explains briefly the concerned routing algorithms. Section 4 is devoted to the discussion of the simulation package. Section 5 presents the results obtained. Section 6 includes the conclusion.

\section{The switching techniques}

The two basic data switching techniques are: the circuit and the store-and-forward switching. The former type is easy to implement, yet its reliability is rather bad and suits better long messages [3]. The second technique uses the idea of store-and-forward in which transmitted data work its way through the network, from channel to channel, queuing at specific nodal points. Two methods are commonly used: the message and the Packet Switching (PS). Both are similar except that in the former method each message is handled in its complete form irrespective of its length. On the other hand, in PS long messages are segmented into shorter packets of specified maximum length. Packets belonging to a message are reassembled into the initial message at the destination node. In either method, a message (in complete form or in shorter packets) would have a header (xo) carrying its identifying information. These networks introduce buffering or queuing delay which plays a critical role in their design. Thus, the switching technique that suits a network may not suit another. All depends on the nature of the different parameters of the network.

Modern time switching and time multiplexing technology allow both modes of operation to be incorporated in one system. One of these techniques, as mentioned above, is the Virtual Cut-through (VC) switching.

\section{The routing algorithms}

The simulation package encounters, the previously mentioned, five advanced adaptive routing algorithms: the SP, the LA, the DR, the DC and the RP. All these algorithms except, the first one, employ the traffic bifurcation technique but they are totally distinct in regard to their control feature, principle of operation and initialization procedure.

SP assigns the physical shortest path for all transmitted packets. It is a centralized regime depending on global information to minimize the network overall average delay, satisfying a network global function. This algorithm is included as a sample for the centralized adaptive routing techniques. Also, it is needed as an initial step in some of the other algorithms [4].
LA uses a distributed policy, the learning automata collective behavior model, to solve the routing problem depending on local information to optimize a user function, the equalization of the retransmission probability on all emanating links at each node [2] and [5].

$\mathrm{DR}$ is also a distributed policy using the deterministic rule to generate the routing decisions at each node depending on centralized information with the objective of optimizing a system or network function, the average delay [16].

DC is mainly a stochastic algorithm depending on local information to satisfy a user optimization, the message incremental delay, through a distributed control policy and distributed computation policy [6].

RP is exactly like DC except that it uses the electrical network analogy to simplify the optimization problem calculations using the relaxation method for computation and optimizing system function, the average delay [14].

For more information about the routing algorithms and their performance see [1], [2], [4], and [10].

\section{Simulation package}

The simulation package is implemented modularly using a group of modules and subprograms written in FORTRAN Language (F77). The package is equally applicable for any network construction, type, or topology, Also, it has been successfully applied, with some minor modifications, for local area networks. The modules of the virtual cut-through have been tested separately, then adopted in the package main program. The package has to be supplied with the network and control data. The network data include the topology, traffic or load model, store-and-forward buffers, and links channels and capacities. The control data include the chosen routing algorithm and its parameters (if applicable), expectations for topology changing, host and node time outs, expected time limit for the simulation experiment and optional trace data and type (if required). The optional traces could be one of: message, special event, or full trace. These traces are very useful in studying the routing algorithms performance under different load models or network conditions. The package has been allowed to run under the two switching techniques and the results together with the different traces have been thoroughly investigated.

The package in this way is practically feasible by allowing any newly developed module to be adopted in the proper place and activated with the appropriate switch. All simulation package aspects are being exposed to long experimentations, extremely valid in both system and structural concepts. The input data characterization is concerned and the simulation structure, together with its 
modular behavior are thoroughly tested. According to these tests the simulator is supplied with control, branching and stop switches. These switches (if activated) issue helping messages, ask for information or show the expected error source. Thus, all the simulation features help describing every structural aspect or input parameter (for the routing algorithms or the switching techniques) throughout the experiments. For more information see [1], [11] and [15].

\section{Results}

The sample network consists of 10 nodes and 24 links. In the simulations performed the delays encountered by each packet are recorded and their average, over the whole simulation period (which is subdivided into time steps of 4 sec. each and epochs of 10 time steps each) are calculated for both switching techniques. The time step and epoch values have been experimentally calculated by the simulator.

The different routing algorithms are applied one after the other and the following parameters are investigated:

\subsection{The average time delay $(T)$}

The delays encountered by each packet are recorded and their averages, over all the experiment time, are calculated and plotted in absolute time compared to one time step (one time step $=4 \mathrm{sec}$ ). Referring to figure (1), it is clear that the DR algorithm gives the best $T$ values in both PS and VC switching techniques. The LA algorithm comes directly after it with a very slight difference. On the other hand, the RP algorithm has the best reduction in delay that moves it before the SP algorithm. DC comes later with the highest delay and less reduction [1]. Generally, all the algorithms stabilize faster in VC technique.

\subsection{The transit time $(T a)$}

Related to the packet delay is the average transit time or the node-to-node network response time (Ta) [15]. This measure, which is useful from the user point of view, is provided by all simulation experiments. Originally, the simulator gives very large values for $T \mathbf{a}$ in VC compared to PS technique, that is due to the average number of crossed nodes (hops $\mathrm{n}$ ). To have common comparison basis we have to divide these large values by $n$. Figure (2) shows that, the SP algorithm is absolutely the best in both techniques. The DR and LA algorithms compare favorably without improvement in the stabilized value of $T \mathbf{a}$ in case of VC technique. On the other hand, the RP algorithm (due to its relaxation nature) has the best improvement in $T a$ values in $\mathrm{VC}$ technique. Finally, the DC algorithm comes the last.

\subsection{The retransmission probability $(R p)$}

The average values of the retransmitted messages or packets, averaged over all the experiment time at average network load of $7200 \mathrm{message} / \mathrm{hr} /$ node are observed. The retransmitted packets are compared with the useful and the total transmitted packets. This illustrates better the absolute and relative $\boldsymbol{R} \boldsymbol{p}$ values. From figure (3), it is noted that $\boldsymbol{R p}$ oscillates initially in the PS technique, while these oscillations damp drastically in the VC technique. However, they all converge in a comparable time except the DC algorithm. For the LA algorithm, $\boldsymbol{R} \boldsymbol{p}$ being the objective function to be optimized, has the minimum values for both switching techniques. While the $\mathrm{RP}$ algorithm gives the highest values for $\boldsymbol{R} \boldsymbol{p}$. In the SP algorithm, it is slightly better. Finally, the DR algorithm converges very fast and gives a reasonably good $\boldsymbol{R} \boldsymbol{p}$ value. This means that distributed policies and/or traffic bifurcation feature improve $\boldsymbol{R} \boldsymbol{p}$ performance in both techniques.

\subsection{The network utilization $(U z)$}

It is a very useful measure specially in the star topology. $U z$ simply specifies how much time the routing algorithm uses the network resources to perform its control and exchange the necessary information. The main resources taken into account are the channel transmission time and the node processing time. Although the node processing time is very small compared to the transmission time, yet it can not be neglected specifically for large values of $\mathrm{n}$ in VC technique. From figure (4), it is noted that the RP algorithm is the best one in both switching techniques with no major difference. Then, comes the SP, the LA and the DR algorithms with a very slight difference between PS and VC techniques. Finally, comes the DC algorithm with the largest value of $U z$. Most of this time is exhausted in the incremental delay algorithm calculations. The large improvement in the SP algorithm is due to the fact that although it is a centralized policy but it does not depend on much frequent data exchange. Also, it is noted that as utilization increases or decreases, the extreme values of $\boldsymbol{U} z, \mathrm{VC}$ technique does not improve its values.

Tables (1) and (2) summarize the previous results. The total weight gives the sum for each algorithm orders. This measure gives just a rather vague indication of the algorithm positions with respect to each other. The LA and the DR algorithms are equal in both switching 
techniques, then comes the SP algorithm with better position in PS technique. After that comes the RP algorithm with the better position in VC technique. Finally, comes the DC algorithm in both switching techniques.

Table (1) Comparison Table in PS

\begin{tabular}{llllll}
\hline $\begin{array}{l}\text { Function } \\
\begin{array}{l}\text { Routing } \\
\text { Algorithm }\end{array}\end{array}$ & Ta & $\boldsymbol{R p}$ & $\boldsymbol{U} \boldsymbol{z}$ & Weight \\
\cline { 1 - 5 } LA & 2nd & 3rd & 1st & 3rd & 9 \\
SP & 3rd & 1st & 4th & 2nd & 10 \\
DR & 1st & 2nd & 2nd & 4th & 9 \\
RP & 4th & 4th & 5th & 1st & 14 \\
DC & 5th & 5th & 3rd & 5th & 18 \\
\end{tabular}

Table (2) Comparison Table in VC

\begin{tabular}{llllll}
\hline $\begin{array}{l}\text { Function } \\
\text { Routing } \\
\text { Algorithm }\end{array}$ & \multicolumn{1}{c}{$\boldsymbol{T a}$} & $\boldsymbol{R p}$ & $\boldsymbol{U} z$ & Weight \\
\hline LA & 2nd & 3rd & 1st & 3rd & 9 \\
SP & 4th & 1st & 4th & 2nd & 11 \\
DR & 1st & 2nd & 2nd & 4th & 9 \\
RP & 3rd & 4th & 5th & 1st & 13 \\
DC & 5th & 5th & 3rd & 5th & 18 \\
& & & & & \\
\end{tabular}

\section{Conclusion}

The paper presents the performance comparison of five routing algorithms under similar load conditions and network topology for PS and VC switching techniques. It provides an insight into the behavior of each routing algorithm as it reflects the network performance parameters. Also, it presents the behavior of the routing algorithms under similar load conditions and network topology for both switching techniques to determine the boundary between the areas of operation which are more attractive for each technique.

The results, illustrated by both curves and tables, show that the LA algorithm gives the best values for the retransmission probability, and it compares favorably for the average delay and transit time with respect to the other algorithms. While the DC algorithm gives the worst values in almost all performance parameters. Finally, the SP algorithm as an example for the centralized algorithms takes the first place (as expected) only in the transit time. Thus, if the distributed algorithm is highly efficient it should be better than the centralized one.

Thus, depending on the performance measure to be optimized and the switching technique to be enforced one can adopt the convenient routing algorithm.

\section{References}

[1] AYAD, N.M.A. and MOHAMED, F.A. "Adaptive Routing Simulation Package - A Comparative Study", Ein Shams Pulletin no. 18, vol. Elec. Eng., 1986, pp. 185-206.

[2] AYAD, N.M.A., MOHAMED, F.A., MADKOUR, M.A. and GHONAIMY, M.A.R. "Using a Learning Automata Collective Behavior Model in Developing an Adaptive Routing Algorithm", Monastir (Tunisia), Vol. 18 Nov. 1985.

[3] BROOMELL, G. and HEATH, J.R. "Classification Categories and Historical Development of Circuit Switching Topologies", ACM computing surveys, vol. 15 no. 2, June 1983, pp. 95-133.

[4] DREYFUS, S.E. "An Appraisal of Some Shortest Path Algorithms", Operation Research, Vol. 17, May-June 1969, pp. 395-412.

[5] EL-FATTAH, Y. M. "Stochastic Automata Modeling of Certain Problems of Collective Behavior", IEEE Trans. On Sys., Man and Cyb., Vol. SMC-10, no. 6, June 1980, pp. 304-314.

[6] GALLAGER, R.G. " Minimum Delay Routing Algorithm Using Distributed Computation", IEEE Trans. On Comm., Vol. COM-25 no. 1, Jan. 1977, pp. 73-85.

[7] GERLA, M. "New Line Tariffs and Their Impact on Network Design", Proc. IAFPS Nat. Conf., vol. 43, May 6-10, 1974, pp. 577-582.

[8] ILYAS, M. and MOUFTAH, H.T. "Delay Analysis of a Modified Cut-through Switching for Multipacket Messages", IEEE Proc., vol. 132, no. 1, Jan. 1985, pp. 45-48.

[9] KERMANI, P. and KLEINROCK, L. "Virtual Cutthrough: A New Computer Communication Switching technique", Computer Networks, vol. 3, 1979, pp. 267-286.

[10] KLEINROCK, L. "Queuing Systems vol. II- Computer Applications", John-Wiley, New York, 1976.

[11] POOLE, T.G. and SZYMANKIEWICZ, J.S. "Using Simulation to Solve Problems", Mc Graw-Hil, 1977.

[12] SCHWARTZ, M. "Computer Communication Network Design and Analysis", Printce-Hall, 1977.

[13] SEIDLER, J. "Principles of Computer Communication Network Design", Ellis Horwood Limited, 1983.

[14] STERN, T. " A Class of Decentralized Routing Algorithms Using Relaxation", IEEE Trans. On Comm., Vol. COM-25, no. 10, Oct. 1977, pp. 1092-1102.

[15] YOUN, Y.S. and UN, C.K. "Performance Analysis of an Integrated Voice/Data Cut-through Switching Network", Computer Networks and ISDN Systems, vol. 21, 1991, pp. 4151.

[16] YUM, T.P. "The Design and Analysis of a Semidynamic Deterministic Routing Rule", IEEE Trans. On Comm., Vol. COM-29, no. 4, April 1981, pp. 43-59. 

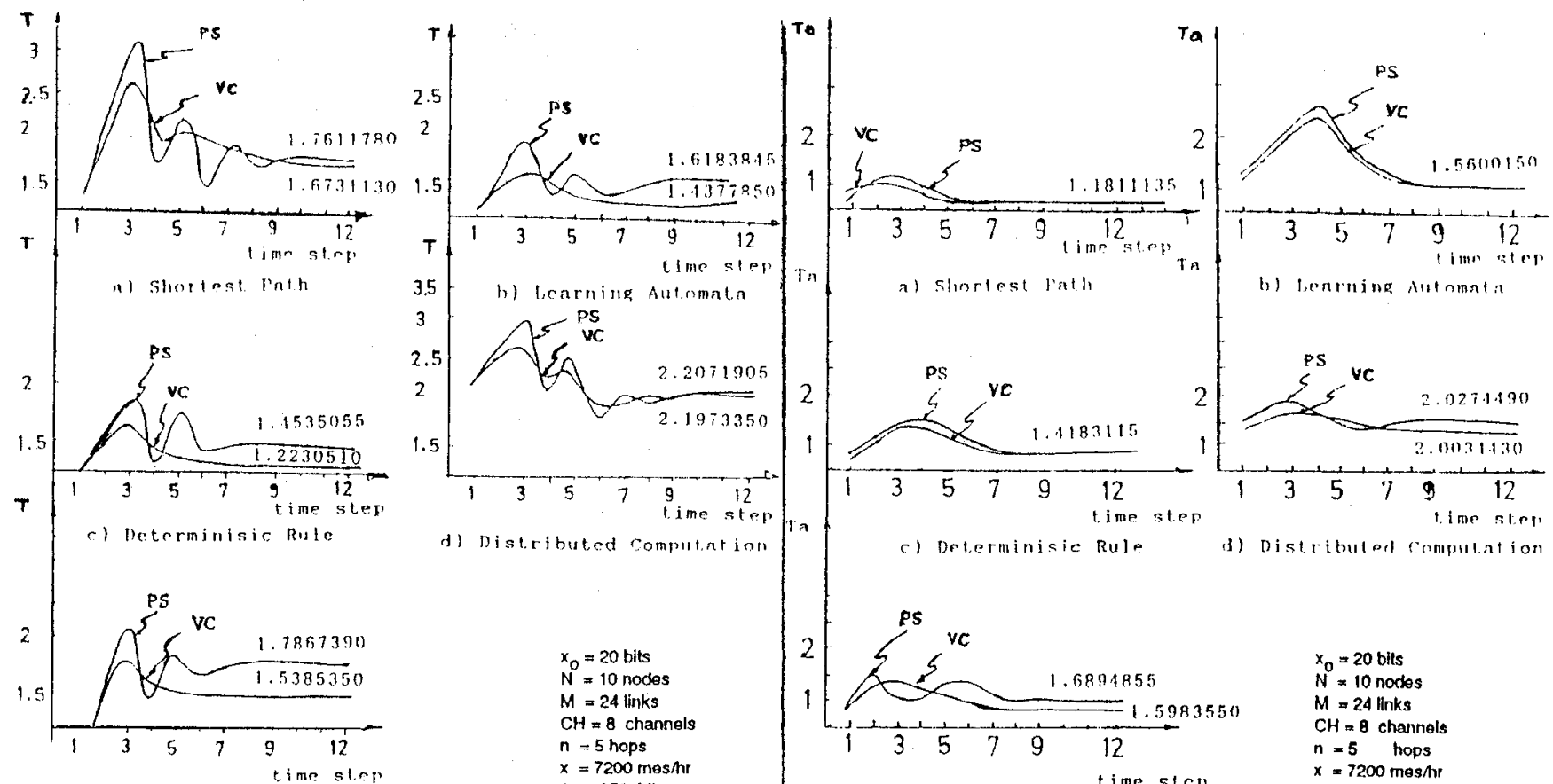

d) Distributed commutation

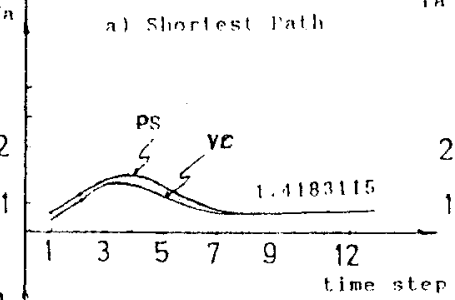

b) ferroing Automatn

c) Determinisic Rule
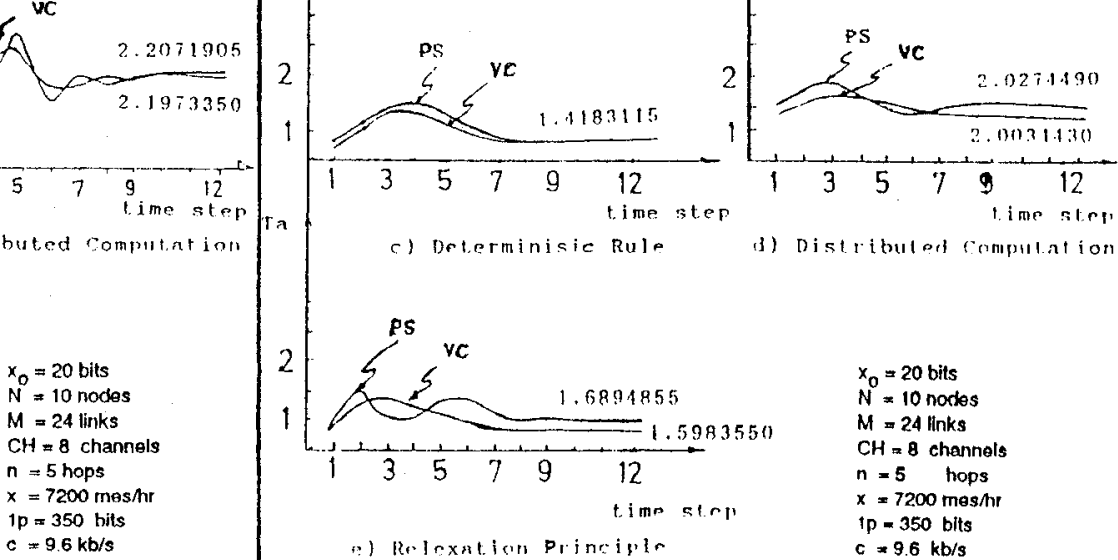

a) Relexation pijacisur

$c=9.6 \mathrm{~kb} / \mathrm{s}$

Fig. 2. The Transit Time Ta

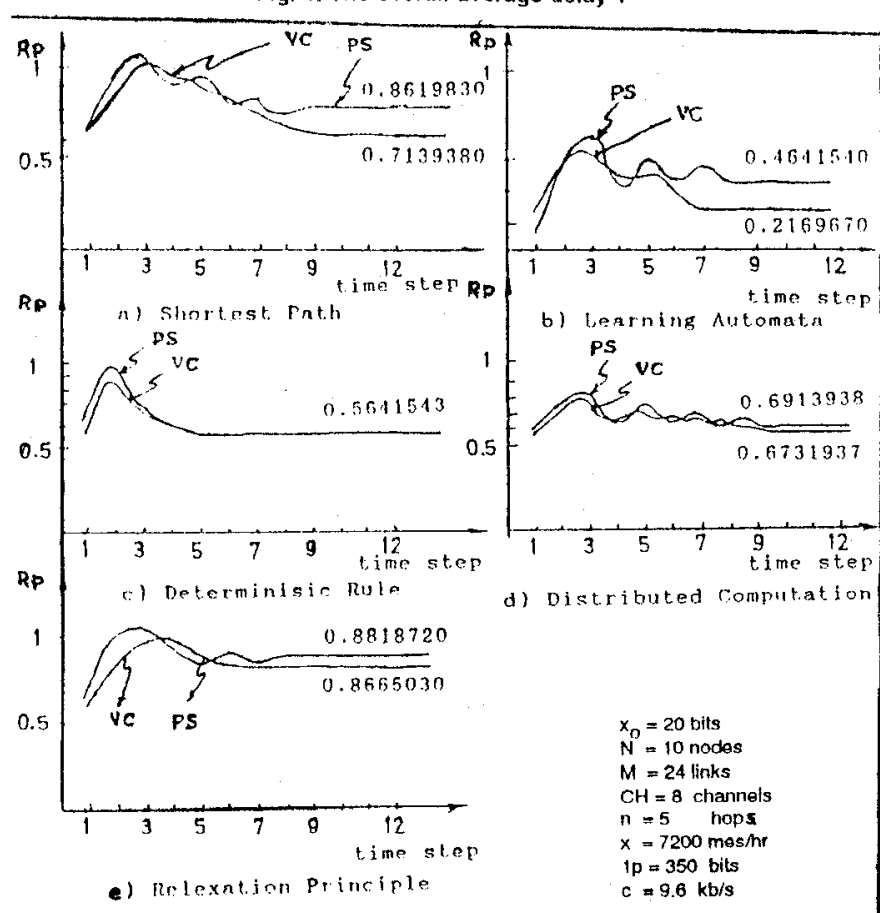

Flg. 3. The Retransmission Probability Rp

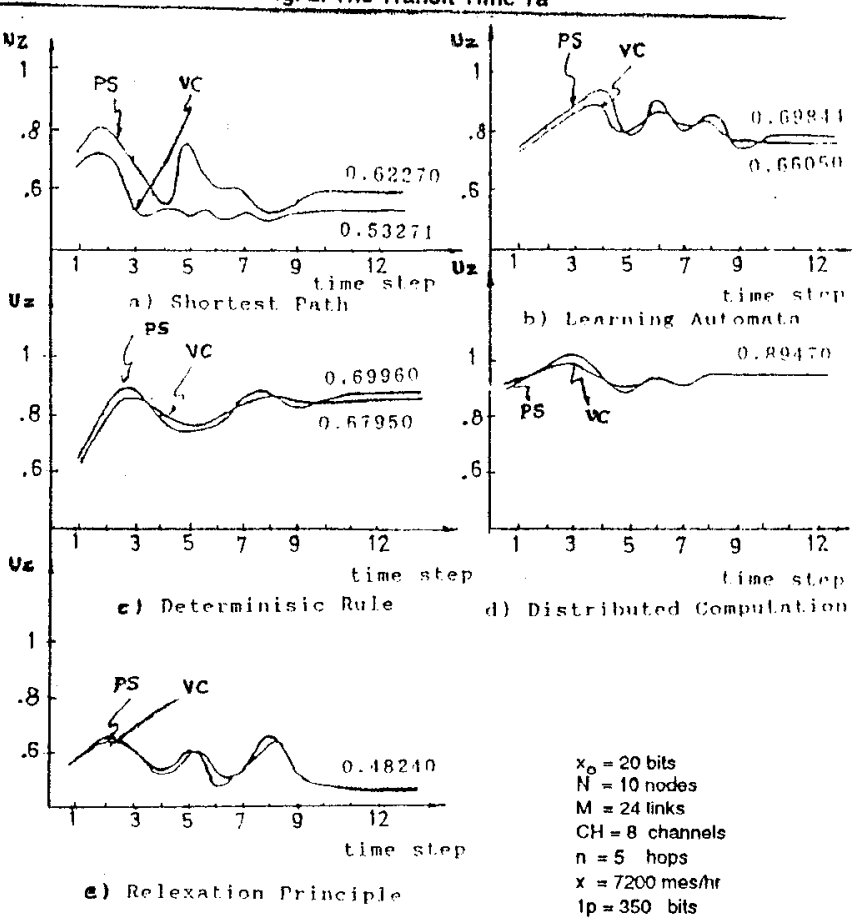

Fig. 4. The Network Utilization Uz 